Article

\title{
Approximation Properties of Solutions of a Mean Value-Type Functional Inequality, II
}

\author{
Soon-Mo Jung ${ }^{1, *,+}$ (D) Ki-Suk Lee ${ }^{2, \dagger}$, Michael Th. Rassias ${ }^{3, \dagger}$ and Sung-Mo Yang ${ }^{2, \dagger}$ \\ 1 Mathematics Section, College of Science and Technology, Hongik University, Sejong 30016, Korea \\ 2 Department of Mathematics Education, Korea National University of Education, Cheongju 28173, Korea; \\ ksleeknue@gmail.com (K.-S.L.); didtjdah2002@hanmail.net (S.-M.Y.) \\ 3 Institute of Mathematics, University of Zurich, CH-8057 Zurich, Switzerland; michail.rassias@math.uzh.ch \\ * Correspondence: smjung@hongik.ac.kr \\ + These authors contributed equally to this work.
}

Received: 22 July 2020; Accepted: 5 August 2020; Published: 6 August 2020

check for updates

\begin{abstract}
Let $X$ be a commutative normed algebra with a unit element $e$ (or a normed field of characteristic different from 2), where the associated norm is sub-multiplicative. We prove the generalized Hyers-Ulam stability of a mean value-type functional equation, $f(x)-g(y)=(x-y) h(s x+t y)$, where $f, g, h: X \rightarrow X$ are functions. The above mean value-type equation plays an important role in the mean value theorem and has an interesting property that characterizes the polynomials of degree at most one. We also prove the Hyers-Ulam stability of that functional equation under some additional conditions.
\end{abstract}

Keywords: Hyers-Ulam stability; Hyers-Ulam-Rassias stability; generalized Hyers-Ulam stability; mean value-type functional equation

MSC: 39B82; 39B62; 39B52; 46J99

\section{Introduction}

In 1940, Ulam gave a lecture on several unresolved important problems at a math seminar at the University of Wisconsin, and in 1960, about two decades later, Ulam published a book [1] detailing the topics covered in the lecture. The following question about the stability of group homomorphism is described in a relatively specific manner in the book:

Assume that $G_{1}$ is a group and $G_{2}$ is a metric group equipped with the metric $d(\cdot, \cdot)$. Given an arbitrary constant $\varepsilon>0$, can we choose a constant $\delta>0$ such that for every function $h: G_{1} \rightarrow G_{2}$ satisfying $d(h(x y), h(x) h(y))<\delta$ for all $x, y \in G_{1}$ there exists a group homomorphism $H: G_{1} \rightarrow G_{2}$ with $d(h(x), H(x))<\varepsilon$ for all $x \in G_{1}$ ?

Just in the next year, Hyers [2] partially solved Ulam's problem for the approximate additive functions in the case of both $G_{1}$ and $G_{2}$ being Banach spaces. Indeed, a problem of mathematical "stability" for the case of the additive functional equation was investigated. It was proved that if a function $f: G_{1} \rightarrow G_{2}$ of a vector space $G_{1}$ into a Banach space $G_{2}$ satisfies the inequality $\|f(x+y)-f(x)-f(y)\| \leq \varepsilon$ for some $\varepsilon>0$ and for all $x, y \in G_{1}$, then there exists an additive function $a: G_{1} \rightarrow G_{2}$ such that $\|f(x)-a(x)\| \leq \varepsilon$ for any $x \in G_{1}$.

Considering these historical backgrounds, the additive Cauchy functional equation $f(x+y)=$ $f(x)+f(y)$ is said to have (or satisfy or meet) the Hyers-Ulam stability in the class of functions $f: G_{1} \rightarrow G_{2}$. This terminology is also applicable for other functional equations. Readers who want to know a more accurate definition of such a terminology should refer to [3-7]. 
The following functional equation

$$
f(x)-g(y)=(x-y) h(x+y)
$$

plays an important role in the mean value theorem and has an interesting property that characterizes the polynomials of degree one and two. Aczél proposed this functional equation in 1963 and then Haruki independently studied it (see [8,9]). We summarize the result of Aczél in the following theorem:

Theorem 1. ([8,9]) Assume that $X$ is a field of characteristic different from 2. The functions $f, g, h: X \rightarrow X$ satisfy the functional Equation (1) for any $x, y \in X$ if and only if there exist $a, b, c \in X$ such that $f(x)=$ $g(x)=a x^{2}+b x+c$ and $h(x)=a x+b$ for any $x \in X$.

In Section 3, we will prove the generalized Hyers-Ulam stability of the functional equation

$$
f(x)-g(y)=(x-y) h(s x+t y)
$$

where $s$ and $t$ are fixed nonzero scalar parameters. In addition, we prove the Hyers-Ulam stability of the functional Equation (2) under some additional condition.

We note that this functional Equation (2) is introduced in detail in book [10] and that this equation is a significant extension of (1).

\section{Preliminaries}

We notice that the generalization of the functional equation (1) was handled by Kannappan, P. K. Sahoo and Jacobson [11] (see also ([10], Theorem 2.5)).

In this section, we will introduce the result of a recent study on the stability problem of an extended form of functional Equation (1).

From now on, we assume that $X$ is a commutative normed algebra over $\mathbb{K}$ with a unit element $e$ (or a normed field of characteristic different from 2), where $\mathbb{K}$ is assumed to be either $\mathbb{R}$ or $\mathbb{C}$. We recommend readers to refer to ([12], Chapter 10) for a detailed definition of normed algebra.

As usual, we assume throughout this paper that the space $X$ is equipped with the sub-multiplicative norm $\|\cdot\|$, i.e., the norm satisfies the following inequality

$$
\|x y\| \leq\|x\|\|y\|
$$

for all $x, y \in X$, which causes the multiplication to be a continuous operation in $X$.

For a given function $\varphi: X \times X \rightarrow[0, \infty)$, we set

$$
\begin{aligned}
\Phi_{s}(x, y)= & \varphi\left(\frac{1}{2 s}(x+y), \frac{1}{2 s}(x-y)\right)+2 \varphi\left(\frac{1}{2 s}(x+y), 0\right) \\
& +\varphi\left(\frac{1}{2 s}(x+y), \frac{1}{2 s}(y-x)\right)+\varphi\left(\frac{1}{2 s}(y-x), \frac{1}{2 s}(x-y)\right) \\
& +\varphi\left(\frac{1}{2 s}(y-x), 0\right)+2 \varphi\left(0, \frac{1}{2 s}(x-y)\right)+\varphi\left(0, \frac{1}{2 s}(y-x)\right)
\end{aligned}
$$

for all $x, y \in X$, where $s$ is a nonzero scalar parameter.

For the case when $s=t$, the generalized Hyers-Ulam stability of the functional Equation (2) was investigated in a recent paper [13], as we see in the following theorem.

Theorem 2. Assume that a function $\varphi: X \times X \rightarrow[0, \infty)$ satisfies the condition

$$
\lim _{n \rightarrow \infty} \frac{1}{2^{n}} \Phi_{s}\left(x, 2^{n} e\right)=0
$$


for any $x \in X$. If functions $f, g, h: X \rightarrow X$ satisfy the inequality

$$
\|f(x)-g(y)-(x-y) h(s x+s y)\| \leq \varphi(x, y)
$$

for all $x, y \in X$, then there exist uniquely determined $a, b \in X$ such that

$$
\begin{gathered}
\left\|f(x)-s a x^{2}-b x-g(0)\right\| \leq \varphi(x, 0), \\
\left\|g(x)-s a x^{2}-b x-f(0)\right\| \leq \varphi(0, x), \\
h(x)=a x+b
\end{gathered}
$$

for all $x \in X$.

We mention that we were able to prove the main theorem of this paper by getting idea and motivation from the proof of Theorem 2.

\section{Generalized Hyers-Ulam Stability of (2)}

It is well known that the stability theorem of Hyers has been generalized for the additive functional equation by including the case when the set of Cauchy differences is not bounded (see [14,15]). In this section, we generalize the ideas from $[7,13-15]$ and prove a general theorem on the stability of functional Equation (2).

Let $X$ be a commutative normed algebra over $\mathbb{K}$ with a unit element $e$ (or a normed field of characteristic different from 2 ), where $\mathbb{K}$ is either $\mathbb{R}$ or $\mathbb{C}$. Moreover, we assume that the space $X$ is equipped with the sub-multiplicative norm $\|\cdot\|$.

Suppose $\varphi: X \times X \rightarrow[0, \infty)$ is a function. For all $x, y \in X$, we will use the following notation

$$
\begin{aligned}
\Phi_{s, t}(x, y)= & \varphi\left(\frac{1}{2 s}(x+y), \frac{1}{2 t}(x-y)\right)+\varphi\left(\frac{1}{2 s}(x-y), \frac{1}{2 t}(x+y)\right) \\
& +\varphi\left(\frac{1}{2 s}(x+y), \frac{1}{2 t}(y-x)\right)+\varphi\left(\frac{1}{2 s}(y-x), \frac{1}{2 t}(x+y)\right) \\
& +\varphi\left(\frac{1}{2 s}(x-y), \frac{1}{2 t}(y-x)\right)+\varphi\left(\frac{1}{2 s}(y-x), \frac{1}{2 t}(x-y)\right) \\
& +2 \varphi\left(\frac{1}{2 s}(x+y), 0\right)+2 \varphi\left(\frac{1}{2 s}(x-y), 0\right) \\
& +2 \varphi\left(\frac{1}{2 s}(y-x), 0\right)+2 \varphi\left(0, \frac{1}{2 t}(x+y)\right) \\
& +2 \varphi\left(0, \frac{1}{2 t}(x-y)\right)+2 \varphi\left(0, \frac{1}{2 t}(y-x)\right),
\end{aligned}
$$

where $s$ and $t$ are nonzero scalar parameters. Moreover, for any fixed $s, t \in \mathbb{K} \backslash\{0\}$, we assume that

$$
\begin{cases}\lim _{n \rightarrow \infty}\left\|r^{-n}\right\| \Phi_{s, t}\left(x, r^{n} x\right)=0 & (\text { for }\|r\|>1), \\ \lim _{n \rightarrow \infty}\left\|r^{-n}\right\|=0=\lim _{n \rightarrow \infty}\left\|r^{-n}\right\| \varphi\left(x, r^{n} y\right) & (\text { for }\|r\|>1), \\ \lim _{n \rightarrow \infty}\left\|r^{n}\right\| \Phi_{s, t}\left(x, r^{-n} x\right)=0 & (\text { for } 0<\|r\|<1)\end{cases}
$$

for all $r, x, y \in X$, where $r^{-1}$ denotes the multiplicative inverse of $r$.

The proof of the following theorem is strongly based on the proof of ([13], Theorem 2.1) or ([7], Theorem 2) or ([10], Theorem 2.3). 
Theorem 3. Assume that $X$ is a commutative normed algebra over $\mathbb{K}$ with a unit element e and every element of $X \backslash\{0\}$ is invertible (or assume that $X$ is a normed field of characteristic different from 2), where the norm is sub-multiplicative and $\mathbb{K}$ is either $\mathbb{R}$ or $\mathbb{C}$. Suppose $s$ and t are fixed nonzero scalar parameters satisfying $s^{2} \neq t^{2}$. Assume that $\|e\|=1$ and a function $\varphi: X \times X \rightarrow[0, \infty)$ satisfies the conditions in (3). If functions $f, g, h: X \rightarrow X$ satisfy the inequality

$$
\|f(x)-g(y)-(x-y) h(s x+t y)\| \leq \varphi(x, y)
$$

for all $x, y \in X$, then there exists a unique element $b$ of $X$ such that

$$
\|f(x)-b x-g(0)\| \leq \varphi(x, 0), \quad\|g(x)-b x-f(0)\| \leq \varphi(0, x), \quad h(x)=b
$$

for all $x \in X$.

Proof. Replacing $y$ in (4) by 0 yields

$$
\|f(x)-x h(s x)-g(0)\| \leq \varphi(x, 0)
$$

for each $x \in X$. Similarly, putting $x=0$ in (4), we get

$$
\|g(y)-y h(t y)-f(0)\| \leq \varphi(0, y)
$$

for all $y \in X$. Furthermore, it follows from (4), (6) and (7) that

$$
\begin{aligned}
& \|x h(s x)-y h(t y)-(x-y) h(s x+t y)-f(0)+g(0)\| \\
& \quad \leq\|f(x)-g(y)-(x-y) h(s x+t y)\|+\|x h(s x)-f(x)+g(0)\|+\|g(y)-y h(t y)-f(0)\| \\
& \quad \leq \varphi(x, y)+\varphi(x, 0)+\varphi(0, y)
\end{aligned}
$$

for all $x, y \in X$. For any element $c$ of $X$, whenever $h$ satisfies the last inequality, so does $h+c$. Hence, we may assume that $h(0)=0$.

If we replace $x$ and $y$ in the last inequality with $\frac{1}{s} x$ and $\frac{1}{t} y$, respectively, then we have

$$
\begin{aligned}
& \left\|\frac{1}{s} x h(x)-\frac{1}{t} y h(y)-\left(\frac{1}{s} x-\frac{1}{t} y\right) h(x+y)-f(0)+g(0)\right\| \\
& \quad \leq \varphi\left(\frac{1}{s} x, \frac{1}{t} y\right)+\varphi\left(\frac{1}{s} x, 0\right)+\varphi\left(0, \frac{1}{t} y\right)
\end{aligned}
$$

for all $x, y \in X$. If we interchange $x$ and $y$ in (8), then we have

$$
\begin{aligned}
& \left\|\frac{1}{s} y h(y)-\frac{1}{t} x h(x)-\left(\frac{1}{s} y-\frac{1}{t} x\right) h(x+y)-f(0)+g(0)\right\| \\
& \quad \leq \varphi\left(\frac{1}{s} y, \frac{1}{t} x\right)+\varphi\left(\frac{1}{s} y, 0\right)+\varphi\left(0, \frac{1}{t} x\right)
\end{aligned}
$$

for any $x, y \in X$.

Subtracting the left terms of (8) and (9) yields

$$
\begin{aligned}
\|x h(x)-y h(y)-(x-y) h(x+y)\| \\
\leq|\tau|\left[\varphi\left(\frac{1}{s} x, \frac{1}{t} y\right)+\varphi\left(\frac{1}{s} y, \frac{1}{t} x\right)+\varphi\left(\frac{1}{s} x, 0\right)\right. \\
\left.\quad+\varphi\left(\frac{1}{s} y, 0\right)+\varphi\left(0, \frac{1}{t} x\right)+\varphi\left(0, \frac{1}{t} y\right)\right]
\end{aligned}
$$

where we set $\tau=\frac{s t}{s+t}$. 
By replacing $y$ in (10) with $-y$, we get

$$
\begin{aligned}
\|x h(x)+y h(-y)-(x+y) h(x-y)\| \\
\leq|\tau|\left[\varphi\left(\frac{1}{s} x, \frac{-1}{t} y\right)+\varphi\left(\frac{-1}{s} y, \frac{1}{t} x\right)+\varphi\left(\frac{1}{s} x, 0\right)\right. \\
\left.\quad+\varphi\left(\frac{-1}{s} y, 0\right)+\varphi\left(0, \frac{1}{t} x\right)+\varphi\left(0, \frac{-1}{t} y\right)\right]
\end{aligned}
$$

for every $x, y \in X$.

On the other hand, if we set $y=-x$ in (10), then we get

$$
\begin{array}{r}
\|x h(x)+x h(-x)\| \leq|\tau|\left[\varphi\left(\frac{1}{s} x, \frac{-1}{t} x\right)+\varphi\left(\frac{-1}{s} x, \frac{1}{t} x\right)+\varphi\left(\frac{1}{s} x, 0\right)\right. \\
\left.+\varphi\left(\frac{-1}{s} x, 0\right)+\varphi\left(0, \frac{1}{t} x\right)+\varphi\left(0, \frac{-1}{t} x\right)\right]
\end{array}
$$

for all $x, y \in X$.

By (10)-(12), we have

$$
\begin{aligned}
& \|(x+y) h(x-y)-(x-y) h(x+y)\| \\
& \leq\|-x h(x)-y h(-y)+(x+y) h(x-y)\| \\
& +\|x h(x)-y h(y)-(x-y) h(x+y)\|+\|y h(y)+y h(-y)\| \\
& \leq|\tau|\left[\varphi\left(\frac{1}{s} x, \frac{1}{t} y\right)+\varphi\left(\frac{1}{s} y, \frac{1}{t} x\right)+\varphi\left(\frac{1}{s} x, \frac{-1}{t} y\right)+\varphi\left(\frac{-1}{s} y, \frac{1}{t} x\right)\right. \\
& \quad+\varphi\left(\frac{1}{s} y, \frac{-1}{t} y\right)+\varphi\left(\frac{-1}{s} y, \frac{1}{t} y\right)+2 \varphi\left(\frac{1}{s} x, 0\right)+2 \varphi\left(\frac{1}{s} y, 0\right) \\
& \left.\quad+2 \varphi\left(\frac{-1}{s} y, 0\right)+2 \varphi\left(0, \frac{1}{t} x\right)+2 \varphi\left(0, \frac{1}{t} y\right)+2 \varphi\left(0, \frac{-1}{t} y\right)\right]
\end{aligned}
$$

for all $x, y \in X$. Substituting $u=x+y$ and $v=x-y$ in (13), we obtain

$$
\|u h(v)-v h(u)\| \leq|\tau| \Phi_{s, t}(u, v)
$$

for all $u, v \in X$.

For any $r \in X$ with $\|r\|>1$ and $n \in \mathbb{N}$, we set $v=r^{n} u$ in (14). Then we get

$$
\left\|r^{-n} h\left(r^{n} u\right)-h(u)\right\| \leq\left\|v^{-1}\right\|\|u h(v)-v h(u)\| \leq|\tau|\left\|r^{-n}\right\|\left\|u^{-1}\right\| \Phi_{s, t}\left(u, r^{n} u\right) .
$$

If we let $n \rightarrow \infty$ in the last inequality, then it follows from (3) that

$$
h(u)=\lim _{n \rightarrow \infty} r^{-n} h\left(r^{n} u\right)
$$

and from this result, we obtain

$$
h(r u)=r \lim _{n \rightarrow \infty} r^{-(n+1)} h\left(r^{n+1} u\right)=r h(u)
$$

for all $r, u \in X$ with $\|r\|>1$.

Analogously, assume that an element $r$ of $X$ satisfies $0<\|r\|<1$ and $n$ is a positive integer. If we set $v=r^{-n} u$ in (14) (hence $v^{-1}=r^{n} u^{-1}$ ), then we have

$$
\left\|r^{n} h\left(r^{-n} u\right)-h(u)\right\| \leq\left\|v^{-1}\right\|\|u h(v)-v h(u)\| \leq|\tau|\left\|r^{n}\right\|\left\|u^{-1}\right\| \Phi_{s, t}\left(u, r^{-n} u\right) .
$$


In view of (3), if we let $n \rightarrow \infty$ in the last inequality, then we have

$$
h(u)=\lim _{n \rightarrow \infty} r^{n} h\left(r^{-n} u\right)
$$

and hence

$$
h(r u)=r \lim _{n \rightarrow \infty} r^{n-1} h\left(r^{-(n-1)} u\right)=r h(u)
$$

for all $r, u \in X$ with $0<\|r\|<1$.

Let $r \in X$ be given with $\|r\|=1$ and $\alpha \in \mathbb{K}$ with $|\alpha|>1$. If we set $v=\alpha r$, then we have $r=\frac{1}{\alpha} v$. Please note that $\|v\|=|\alpha|>1$ and $0<\left\|\frac{1}{\alpha} e\right\|=\frac{1}{|\alpha|}<1$. It then follows from (15) and (16) that

$$
h(r u)=h\left(\frac{1}{\alpha} v u\right)=h\left(v \frac{1}{\alpha} u\right)=v h\left(\frac{1}{\alpha} e u\right)=\frac{1}{\alpha} e v h(u)=r h(u) .
$$

Since we assumed $h(0)=0$, we may conclude that $h(r u)=r h(u)$ for all $r, u \in X$. If we put $r=x$ and $u=e$, then we have $h(x)=a x$, where we set $a=h(e)$. When $h(0) \neq 0$, we have $h(x)=a x+b$ with $b=h(0)$.

We temporarily assume that $a \in X \backslash\{0\}$. If we substitute the last expression for $h$ into (8) and replace $x$ and $y$ with $s x$ and $t y$, respectively, then we get

$$
|s-t|\|a x y\| \leq \varphi(x, y)+\varphi(x, 0)+\varphi(0, y)+\|f(0)\|+\|g(0)\|
$$

for all $x, y \in X$. In the last inequality, we put $x=a^{-1}, y=r^{n}$ for some $r \in X$ with $\|r\|>1$ and for any $n \in \mathbb{N}$ and we obtain

$$
|s-t|\left\|r^{n}\right\| \leq \varphi\left(a^{-1}, r^{n}\right)+\varphi\left(a^{-1}, 0\right)+\varphi\left(0, r^{n}\right)+\|f(0)\|+\|g(0)\| .
$$

In view of (3), we further have

$$
\begin{aligned}
0<|s-t| & =\lim _{n \rightarrow \infty}|s-t|\left\|r^{-n} r^{n}\right\| \\
& \leq \lim _{n \rightarrow \infty}\left\|r^{-n}\right\|\left(\varphi\left(a^{-1}, r^{n}\right)+\varphi\left(a^{-1}, 0\right)+\varphi\left(0, r^{n}\right)+\|f(0)\|+\|g(0)\|\right) \\
& =0
\end{aligned}
$$

which leads to a contradiction. Therefore, we conclude that $a=0$, i.e.,

$$
h(x)=b .
$$

Finally, in view of (6) and (17), we obtain

$$
\|f(x)-b x-g(0)\| \leq \varphi(x, 0)
$$

for any $x \in X$. Similarly, by (7) and (17), we have

$$
\|g(x)-b x-f(0)\| \leq \varphi(0, x)
$$

for all $x \in X$.

Now we can prove the uniqueness of $b$ as follows. If there exists $b^{\prime} \neq b$ such that

$$
\left\|g(x)-b^{\prime} x-f(0)\right\| \leq \varphi(0, x)
$$


for all $x \in X$, then

$$
\|x\| \leq \frac{2}{\left|b-b^{\prime}\right|} \varphi(0, x)
$$

for any $x \in X$. Taking $x=r^{n} e$ with $\|r\|>1$, it follows that

$$
1=\left\|r^{-n} r^{n}\right\| \leq\left\|r^{-n}\right\|\left\|r^{n}\right\| \leq \frac{2}{\left|b-b^{\prime}\right|}\left\|r^{-n}\right\| \varphi\left(0, r^{n} e\right) \rightarrow 0
$$

as $n \rightarrow \infty$, a contradiction.

In Theorem 3, the difference between $f$ (or $g$ ) and a linear polynomial is bounded by $\varphi(x, 0)$ (or $\varphi(0, x)$ ). Instead of (3), we now assume that

$$
\left\|r^{-1}\right\|<1 \text { if }\|r\|>1
$$

for all $r \in X$. Under this condition (18), we prove the Hyers-Ulam stability of the functional Equation (2).

Corollary 1. Assume that $X$ is a commutative normed algebra over $\mathbb{K}$ with a unit element $e$ and every element of $X \backslash\{0\}$ is invertible (or assume that $X$ is a normed field of characteristic different from 2 ), where the norm is sub-multiplicative. Assume that $\|e\|=1$ and the condition (18) is satisfied. For any fixed $\varepsilon>0$, if functions $f, g, h: X \rightarrow X$ satisfy the inequality

$$
\|f(x)-g(y)-(x-y) h(s x+t y)\| \leq \varepsilon
$$

for all $x, y \in X$, then there exists an element $b$ of $X$ such that

$$
\|f(x)-b x-g(0)\| \leq \varepsilon, \quad\|g(x)-b x-f(0)\| \leq \varepsilon, \quad h(x)=b
$$

for all $x \in X$.

We remark that the Euclidean norm defined on $\mathbb{R}$ is sub-multiplicative. We now introduce a characterization of polynomials of degree one as an application of Theorem 3.

Corollary 2. Let $s$ and $t$ be fixed nonzero real numbers satisfying $s^{2} \neq t^{2}$. Suppose $\varphi: \mathbb{R} \times \mathbb{R} \rightarrow[0, \infty)$ is a function satisfying the conditions in (3). A differentiable function $f: \mathbb{R} \rightarrow \mathbb{R}$ satisfies the inequality

$$
\left|f(x)-f(y)-(x-y) f^{\prime}(s x+t y)\right| \leq \varphi(x, y)
$$

for all $x, y \in \mathbb{R}$ if and only if there exist real numbers $b$ and $c$ such that $f(x)=b x+c$ for all $x \in \mathbb{R}$.

\section{Discussion}

In the paper [13], the generalized Hyers-Ulam stability of the functional Equation (2) was studied for $s=t$. More precisely, if $s=t$, it was shown that $f$ and $g$, which are the approximate solutions of the functional Equation (2), can be estimated by quadratic functions, and $h$ is a linear function.

In the present paper, on the other hand, we studied the generalized Hyers-Ulam stability of the functional Equation (2) when the $s$ and $t$ are arbitrary constants other than 0. Moreover, we have proved in this paper that $f$ and $g$, which are the approximate solutions to the functional Equation (2), can be estimated as linear functions and $h$ is a constant function. These are different from the results in the paper [13].

Author Contributions: Conceptualization, S.-M.J.; writing-original draft preparation, S.-M.J., K.-S.L., M.T.R. and S.-M.Y.; writing-review and editing, S.-M.J., K.-S.L., M.T.R. and S.-M.Y. All authors have read and agreed to the published version of the manuscript. 
Funding: This research received no external funding.

Conflicts of Interest: The authors declare no conflict of interest.

\section{References}

1. Ulam, S.M. Problems in Modern Mathematics; Wiley: New York, NY, USA, 1960; Chapter VI.

2. Hyers, D.H. On the stability of the linear functional equation. Proc. Natl. Acad. Sci. USA 1941, 86, $222-224$. [CrossRef] [PubMed]

3. Forti, G.L. Hyers-Ulam stability of functional equations in several variables. Aequ. Math. 1995, 50, $143-190$. [CrossRef]

4. Hyers, D.H.; Isac, G.; Rassias, T.M. Stability of Functional Equations in Several Variables; Birkhäuser: Berlin, Germany, 1998.

5. Hyers, D.H.; Rassias, T.M. Approximate homomorphisms. Aequ. Math. 1992, 44, 125-153. [CrossRef]

6. Jung, S.-M. Hyers-Ulam-Rassias Stability of Functional Equations in Nonlinear Analysis; Springer: New York, NY, USA, 2011.

7. Jung, S.-M.; Sahoo, P.K. On the stability of a mean value type functional equation. Demonstr. Math. 2000, 33, 793-796. [CrossRef]

8. Aczél, J. A mean value property of the derivative of quadratic polynomials-Without mean values and derivatives. Math. Mag. 1985, 58, 42-45.

9. Haruki, S. A property of quadratic polynomials. Am. Math. Mon. 1979, 86, 577-579. [CrossRef]

10. Sahoo, P.K.; Riedel, T. Mean Value Theorems and Functional Equations; World Scientific: Singapore, 1998.

11. Kannappan Pl Sahoo, P.K.; Jacobson, M.S. A characterization of low degree polynomials. Demonstr. Math. 1995, 28, 87-96.

12. Rudin, W. Functional Analysis, 2nd ed.; McGraw-Hill: New York, NY, USA, 1991.

13. Choi, G.; Jung, S.-M.; Lee, Y.-H. Approximation properties of solutions of a mean value type functional inequalities. J. Nonlinear Sci. Appl. 2017, 10, 4507-4514. [CrossRef]

14. Găvruţa, P. A generalization of the Hyers-Ulam-Rassias stability of approximately additive mappings. J. Math. Anal. Appl. 1994, 184, 431-436. [CrossRef]

15. Rassias, T.M. On the stability of the linear mapping in Banach spaces. Proc. Am. Math. Soc. 1978, 72, $297-300$. [CrossRef]

(C) 2020 by the authors. Licensee MDPI, Basel, Switzerland. This article is an open access article distributed under the terms and conditions of the Creative Commons Attribution (CC BY) license (http:/ / creativecommons.org/licenses/by/4.0/). 